\title{
On a Batch Arrival Queuing System Equipped with a Stand-by Server during Vacation Periods or the Repairs Times of the Main Server
}

\author{
Rehab F. Khalaf, ${ }^{1}$ Kailash C. Madan, ${ }^{2}$ and Cormac A. Lukas ${ }^{1}$ \\ ${ }^{1}$ School of Information Systems Computing and Mathematics, Brunel University, \\ Middlesex UB83PH, UK \\ ${ }^{2}$ College of Information Technology, Ahlia University, P.O. Box 10878, Bahrain
}

Correspondence should be addressed to Rehab F. Khalaf, rihabfadil@gmail.com

Received 25 January 2011; Accepted 20 May 2011

Academic Editor: Rongling Wu

Copyright (c) 2011 Rehab F. Khalaf et al. This is an open access article distributed under the Creative Commons Attribution License, which permits unrestricted use, distribution, and reproduction in any medium, provided the original work is properly cited.

\begin{abstract}
We study a queuing system which is equipped with a stand-by server in addition to the main server. The stand-by server provides service to customers only during the period of absence of the main server when either the main server is on a vacation or it is in the state of repairs due to a sudden failure from time to time. The service times, vacation times, and repair times are assumed to follow general arbitrary distributions while the stand-by service times follow exponential distribution. Supplementary variables technique has been used to obtain steady state results in explicit and closed form in terms of the probability generating functions for the number of customers in the queue, the average number of customers, and the average waiting time in the queue while the MathCad software has been used to illustrate the numerical results in this work.
\end{abstract}

\section{Introduction}

Due to their wide applications in flexible manufacturing or computer communication systems, $M^{[X]} / G / 1$ queueing system with vacations and $M^{[X]} / G / 1$ queueing system with breakdowns have been studied by several authors including [1-8]. Recently the authors of [9] have studied some queueing systems with vacations and breakdowns.

In this work, we study an $M^{[X]} / G / 1$ queueing system with Bernoulli schedule vacations and random breakdowns with an additional significant assumption that the system deploys a stand-by server during the vacation periods and the repair periods of the main server.

Madan [10] studied the steady state behavior of a queuing system with a stand-by server which provides service to costumers only during the repair period. In that work, repair 
times were assumed to follow an exponential distribution. The present paper considers both vacations and breakdowns with additional assumptions of deployment of a standby during the vacation periods and repair periods. We generalize results obtained not only by Madan [10] but also the results obtained by Maraghi et al. [9]. Most importantly, we assume that the service times, vacation times, and repair times have different general (arbitrary) distributions while the stand-by service times follow exponential distribution. Out of five distributions in this model we assume that four are a generally distributed, this is very important because all the other distributions such as exponential, deterministic, and Erlang-k distributions will be included.

The rest of this paper is arranged as follow. Section 2 gives the assumptions underlying the considered queueing system. Related definitions and used notations are given in Section 3. Equations governing the system are formulated in Section 4. In Section 5, we give the solution of the equations formulated in the previous section to find the queue size distribution at a random epoch. The average queue size and the average waiting time are given in Section 6. In Section 7, we consider a numerical example to illustrate application of our results.

\section{Assumptions}

Customers arrive at the system in batches of variable size in a compound Poisson process. Let $\lambda c_{i} \Delta t(i=1,2,3, \ldots)$ be the first order probability that a batch of $i$ customers arrives at the system during a short interval of time $(t, t+\Delta t)$, where $0 \leq c_{i} \leq 1$ and $\sum_{i=1}^{\infty} c_{i}=1$ and $\lambda>0$ is the mean arrival rate of batches. The customers provided service one by one on a "first comefirst served basis."

The service times of the main server follow a general (arbitrary) distribution with distribution function $G(s)$ and density function $g(s)$. Let $\mu(x) \Delta x$ be the conditional probability

density of service completion during the interval $(x, x+\Delta x]$, given that the elapsed service time is $x$, so that

$$
\mu(x)=\frac{g(x)}{1-G(x)}
$$

and, therefore

$$
g(s)=\mu(s) e^{-\int_{0}^{s} \mu(x) d x}
$$

On completion of a service, the server may take a vacation of random length with probability $P$, or may stay in the system providing service with probability $1-P$, where $0 \leq P \leq 1$.

The server's vacation times follow a general (arbitrary) distribution with distribution function $B(v)$ and density function $b(v)$. Let $\beta(x) \Delta x$ be the conditional probability of a completion of a vacation during the interval $(x, x+d x)$ given that the elapsed vacation time is $x$, so that

$$
\beta(x)=\frac{b(x)}{1-B(x)}
$$


and, therefore

$$
b(v)=\beta(v) e^{-\int_{0}^{v} \beta(x) d x} .
$$

The system may break down at random, and breakdowns are assumed to occur according to a Poisson stream with mean breakdown rate $\alpha>0$. Further we assume that once the system breaks down, the customer whose service is interrupted comes back to the head of the queue but it is instantly taken up for service by the stand-by server.

Once the system breaks down, its repairs start immediately and the duration of repairs follows a general (arbitrary) distribution with distribution function $F(r)$ and density function $f(r)$. Let $\gamma(x) \Delta x$ be the conditional probability density of repair completion during the interval $(x, x+\Delta x]$, given that the elapsed repair time is $x$, so that

$$
\gamma(x)=\frac{f(x)}{1-F(x)}
$$

and, therefore

$$
f(r)=\gamma(r) e^{-\int_{0}^{r} \gamma(x) d x}
$$

The stand-by server starts serving the customers as soon as the main server breaks down or as soon as the main server leaves for a vacation after completing a service. The stand-by service times follow an exponential distribution with stand-by service rate $\delta>0$ and mean stand-by service time $1 / \delta$.

We further assume that the main server joins the system immediately after the completion of its vacation or completion of its repairs, and the customer being served by the stand-by server is immediately transferred to the main server to start a service afresh.

All stochastic processes involved in the system are independent of each other.

\section{Notations}

We let

(i) $P_{n}(t, x)$ : probability that at time $t$, there are $n \geq 0$ customers in the queue excluding one customer in the service served by the main server, and the elapsed service time of this customer is $x$. Accordingly, $P_{n}(t)=\int_{0}^{\infty} P_{n}(t, x) d x$ denotes the probability that there are $n \geq 1$ customers in the queue excluding one customer in service irrespective of the value of $x$;

(ii) $V_{n}(t, x)$ : probability that at time $t$, there are $n \geq 0$ customers in the queue (and one customer is being served by the stand-by server), and the main server is on vacation with elapsed vacation time $x$. Accordingly, $V_{n}(t)=\int_{0}^{\infty} V_{n}(t, x) d x$ denotes the probability that at time $t$, there are $n \geq 0$ customers in the queue and the server is on vacation irrespective of the value of $x$. As soon as the vacation starts the stand-by server starts serving the customers in the system; 
(iii) $R_{n}(t, x)$ : Probability that at time $t$, there are $n(n \geq 0)$ customers in the queue (and one customer is being served by the stand-by server) while the system is under repair with elapsed repair time $x$. Accordingly, $R_{n}(t)=\int_{0}^{\infty} R_{n}(t, x) d x$ denotes the probability that at time $t$, there are $n \geq 0$ customers in the queue and the server is under repair irrespective of the value of $x$;

(iv) $Q(t)$ : probability that at time $t$, there are no customers in the system and the server is idle but available in the system.

Assuming that the steady state exists, we let

$$
\begin{gathered}
\lim _{t \rightarrow \infty} A_{n}(t, x)=A_{n}(x), \quad \lim _{t \rightarrow \infty} A_{n}(t)=\lim _{t \rightarrow \infty} \int_{0}^{\infty} A_{n}(t, x) d x=A_{n}, \\
\lim _{t \rightarrow \infty} \frac{d A_{n}(t)}{d t}=0, \quad \text { where } A=P, V, R, \\
\lim _{t \rightarrow \infty} Q(t)=Q .
\end{gathered}
$$

\section{Equations Governing the System}

According to the assumptions mentioned above, we have four possible states of our system during a short time interval $(t, t+\Delta t)$ : the first state is that the main server is providing service, the second is that the main server is on vacation and the stand-by server is providing service, the third state is that the main server is inactive due to a system breakdown and is under repair and the stand-by server is providing service, the last possible state is that the server is idle (there are no customers in the system) but available in the system. By discussing the probabilities of every situation and finding the limit as $\Delta t \rightarrow 0$, we obtain the following set of differential-difference equations

$$
\begin{gathered}
\frac{\partial}{\partial x} P_{n}(x)=-(\lambda+\mu(x)+\alpha) P_{n}(x)+\lambda \sum_{i=1}^{n-1} c_{i} P_{n-i}(x), \quad n \geq 1, \\
\frac{\partial}{\partial x} P_{0}(x)=-(\lambda+\mu(x)+\alpha) P_{0}(x), \\
\frac{\partial}{\partial x} V_{n}(x)=-(\lambda+\beta(x)+\delta) V_{n}(x)+\lambda \sum_{i=1}^{n} c_{i} V_{n-i}(x)+\delta V_{n+1}(x), \quad n \geq 1, \\
\frac{\partial}{\partial x} V_{0}(x)=-(\lambda+\beta(x)+\delta) V_{0}(x)+\delta V_{1}(x), \\
\frac{\partial R_{n}(x)}{\partial x}=-(\lambda+\gamma(x)+\delta) R_{n}(x)+\lambda \sum_{i=1}^{n} c_{i} R_{n-i}(x)+\delta R_{n+1}(x), \quad n \geq 1, \\
\frac{\partial R_{0}(x)}{\partial x}=-(\lambda+\gamma(x)+\delta) R_{0}(x)+\delta R_{1}(x), \\
\lambda Q=\int_{0}^{\infty} R_{0}(x) \gamma(x) d x+(1-p) \int_{0}^{\infty} P_{0}(x) \mu(x) d x+(1-r) \int_{0}^{\infty} V_{0}(x) \beta(x) d x .
\end{gathered}
$$


The following boundary conditions will be used to solve the above equations:

$$
\begin{aligned}
P_{n}(0)= & (1-p) \int_{0}^{\infty} P_{n+1}(x) \mu(x) d x+\int_{0}^{\infty} V_{n+1}(x) \beta(x) d x \\
& +\int_{0}^{\infty} R_{n+1}(x) \gamma(x) d x+\lambda c_{n+1} Q, \quad n \geq 0, \\
V_{n}(0)= & p \int_{0}^{\infty} P_{n}(x) \mu(x) d x, \quad n \geq 0, \\
R_{n}(0)= & \alpha \int_{0}^{\infty} P_{n-1}(x) d x, \quad n \geq 1, \\
R_{0}(0)= & 0 .
\end{aligned}
$$

\section{Queue Size Distribution at a Random Epoch}

Defining the following probability generating functions

$$
\begin{gathered}
A_{q}(x, z)=\sum_{n=0}^{\infty} z^{n} A_{n}(x), \quad A_{q}(z)=\sum_{n=0}^{\infty} z^{n} A_{n}, \\
A=P, V, R, \\
C(z)=\sum_{i=1}^{\infty} z^{i} c_{i}
\end{gathered}
$$

we multiply (4.1) by $z^{n}$, take summation over $n$ from 1 to $\infty$, adding to (4.2) then by simplifying and using (5.1) we get

$$
\frac{\partial}{\partial x} P_{q}(x, z)+(\lambda-\lambda C(z)+\mu(x)+\alpha) P_{q}(x, z)=0 .
$$

Using the same process, from (4.3), (4.4) and (4.5), (4.6) we get, respectively,

$$
\begin{aligned}
& \frac{\partial}{\partial x} V_{q}(x, z)+\left(\lambda-\lambda C(z)+\beta(x)+\delta-\frac{\delta}{z}\right) V_{q}(x, z)=0 \\
& \frac{\partial}{\partial x} R_{q}(x, z)+\left(\lambda-\lambda C(z)+\gamma(x)+\delta-\frac{\delta}{z}\right) R_{q}(x, z)=0 .
\end{aligned}
$$


Multiply (4.8) by $z^{n+1}$, sum over $n$ from 0 to $\infty$, and use the generating functions defined in (5.1), we get

$$
\begin{aligned}
z P_{q}(0, z) & =(1-p) \int_{0}^{\infty} P_{q}(x, z) \mu(x) d x+\int_{0}^{\infty} V_{q}(x, z) \beta(x) d x+\int_{0}^{\infty} R_{q}(x, z) \gamma(x) d x+\lambda C(z) Q \\
& -\left[(1-p) \int_{0}^{\infty} P_{0}(x) \mu(x) d x+(1-r) \int_{0}^{\infty} V_{0}(x, z) \beta(x) d x+\int_{0}^{\infty} R_{0}(x, z) \gamma(x) d x\right] .
\end{aligned}
$$

From (4.7), we have

$$
\begin{aligned}
z P_{q}(0, z) & =(1-p) \int_{0}^{\infty} P_{q}(x, z) \mu(x) d x+\int_{0}^{\infty} V_{q}(x, z) \beta(x) d x+\int_{0}^{\infty} R_{q}(x, z) \gamma(x) d x \\
& +\lambda Q(C(z)-1) .
\end{aligned}
$$

Multiply (4.9) by $z^{n}$ and sum over $n$ from 0 to $\infty$, we get

$$
V_{q}(0, z)=p \int_{0}^{\infty} P_{q}(x, z) \mu(x) d x
$$

Similarly, from (4.10) we get

$$
R_{q}(0, z)=\alpha z \int_{0}^{\infty} P_{q}(x, z) d x=\alpha z P_{q}(z), \quad n \geq 0
$$

Integrating (5.2) from 0 to $x$ yields

$$
P_{q}(x, z)=P_{q}(0, z) e^{-(\lambda-\lambda C(z)+\alpha) x-\int_{0}^{x} \mu(t) d t},
$$

where $P_{q}(0, z)$ is given by (5.6). Let us consider $a=\lambda-\lambda C(z)+\alpha$. Integrating equation (5.9) by parts with respect to $x$ yields

$$
P_{q}(z)=P_{q}(0, z)\left(\frac{1-G^{*}(a)}{a}\right)
$$

where $G^{*}(a)=\int_{0}^{\infty} e^{-a x} d G(x)$ is the Laplace-Stieltjes transform of the service time $G(x)$.

Now multiplying both sides of (5.9) by $\mu(x)$ and integrating over $x$ we get

$$
\int_{0}^{\infty} P_{q}(x, z) \mu(x) d x=P_{q}(0, z) G^{*}(a)
$$


Journal of Probability and Statistics

Using (5.11), from (5.7) we get

$$
V_{q}(0, z)=p P_{q}(0, z) G^{*}(a)
$$

Similarly, we integrate (5.3) from 0 to $x$, we get

$$
V_{q}(x, z)=V_{q}(0, z) e^{-(\lambda-\lambda C(z)+\delta-\delta / x) x-\int_{0}^{x} \beta(t) d t}
$$

Substituting by the value of $V_{q}(0, z)$ from (5.12) in (5.13) we get

$$
V_{q}(x, z)=p P_{q}(0, z) G^{*}(a) e^{-(\lambda-\lambda C(z)+\delta-\delta / z) x-\int_{0}^{x} \beta(t) d t}
$$

Let us consider $b=\lambda-\lambda C(z)+\delta-\delta / z$ now integrating (5.14) by parts with respect to $x$ we get

$$
V_{q}(z)=\frac{p P_{q}(0, z) G^{*}(a)\left(1-B^{*}(b)\right)}{b}
$$

where $B^{*}(b)=\int_{0}^{\infty} e^{-b x} d B(x)$ is the Laplace-Stieltjes transform of the vacation time $B(x)$.

Now multiplying both sides of (5.14) by $\beta(x)$ and integrating over $x$ we get

$$
\int_{0}^{\infty} V_{q}(x, z) \beta(x) d x=p P_{q}(0, z) G^{*}(a) B^{*}(b) .
$$

Now integrating (5.4) from 0 to $x$, yields

$$
R_{q}(x, z)=R_{q}(0, z) e^{-(\lambda-\lambda C(z)+\delta-\delta / z) x-\int_{0}^{x} \gamma(t) d t}
$$

Substituting by the value of $R_{q}(0, z)$ from (5.8) in (5.17) we get

$$
R_{q}(x, z)=\frac{\alpha z P_{q}(0, z)\left(1-G^{*}(a)\right)}{a} e^{-(\lambda-\lambda C(z)+\delta-\delta / z) x-\int_{0}^{x} r(t) d t}
$$

integrating (5.18) by parts with respect to $x$ we get

$$
R_{q}(z)=\alpha z P_{q}(0, z)\left(\frac{\left(1-G^{*}(a)\right)\left(1-F^{*}(b)\right)}{a b}\right)
$$

where $F^{*}(b)=\int_{0}^{\infty} e^{-(\lambda-\lambda C(z)+\delta-\delta / z) x} d F(x)$ is the Laplace-Stieltjes transform of the repair time $F(x)$. 
Now multiplying both sides of (5.18) by $\gamma(x)$ and integrating over $x$ we get

$$
\int_{0}^{\infty} R_{q}(x, z) \gamma(x) d x=\alpha z P_{q}(0, z)\left(\frac{1-G^{*}(a)}{a}\right) F^{*}(b) .
$$

Now using (5.11), (5.16) and (5.20), (5.6) becomes

$$
P_{q}(0, z)=\frac{-a c Q}{a\left(z-G^{*}(a)\left(1-p+p B^{*}(b)\right)\right)-\alpha z\left(1-G^{*}(a)\right) F^{*}(b)}
$$

where $c=\lambda-\lambda C(z)$, from (5.21) equations (5.10), (5.15) and (5.19) become, respectively,

$$
\begin{aligned}
& P_{q}(z)=\frac{-c Q\left(1-G^{*}(a)\right)}{a\left(z-G^{*}(a)\left(1-p+p B^{*}(b)\right)\right)-\alpha z\left(1-G^{*}(a)\right) F^{*}(b)}, \\
& V_{q}(z)=\frac{-a c Q p G^{*}(a)\left(1-B^{*}(b)\right)}{a b\left(z-G^{*}(a)\left(1-p+p B^{*}(b)\right)\right)-\alpha z b\left(1-G^{*}(a)\right) F^{*}(b)}, \\
& R_{q}(z)=\frac{-\alpha z c Q\left(1-G^{*}(a)\right)\left(1-F^{*}(b)\right)}{a b\left(z-G^{*}(a)\left(1-p+p B^{*}(b)\right)\right)-\alpha z b\left(1-G^{*}(a)\right) F^{*}(b)} .
\end{aligned}
$$

Let $S_{q}(z)$ denote the probability generating function of the queue size irrespective of the state of the system. That is, $S_{q}(z)=P_{q}(z)+V_{q}(z)+R_{q}(z)$.

Then adding (5.22) we obtain

$$
S_{q}(z)=\frac{-c Q\left(1-G^{*}(a)\right)\left\{b+\alpha z\left(1-F^{*}(b)\right)\right\}-a c Q p G^{*}(a)\left(1-B^{*}(b)\right)}{a b\left(z-G^{*}(a)\left(1-p+p B^{*}(b)\right)\right)-\alpha z b\left(1-G^{*}(a)\right) F^{*}(b)} .
$$

In order to find $Q$, we use the normalization condition

$$
S_{q}(1)+Q=1
$$

Note that if $z=1$ then $b=0$ and $c=0$, so $S_{q}(1)$ is indeterminate of $0 / 0$ form. Therefore, we apply L'Hopitals Rule twice on (5.23), we get

$$
S_{q}(1)=\lim _{z \rightarrow 1} \frac{N^{\prime \prime}(z)}{D^{\prime \prime}(z)}
$$


where $N(z)$ and $D(z)$ are the numerator and denominator of the right hand side of (5.23) respectively. Double primes in (5.25) denote the second derivative at $z=1$. Carrying out the derivatives at $z=1$ we have

$$
\begin{gathered}
N^{\prime \prime}(1)=-2 Q \lambda E(I)(\lambda E(I)-\delta)\left\{\left(1-G^{*}(\alpha)\right)\{1+\alpha E(R)\}+\alpha p G^{*}(a) E(V)\right\}, \\
D^{\prime \prime}(1)=2(\lambda E(I)-\delta)\left\{\left(1-G^{*}(a)\right)(\lambda E(I)+\alpha\{1+(\lambda E(I)-\delta) E(R)\})\right. \\
\left.-\alpha\left(1-p(\lambda E(I)-\delta) G^{*}(a) E(V)\right)\right\},
\end{gathered}
$$

where $C(1)=1, C^{\prime}(1)=E(I)$ is the mean batch size of the arriving customers, $B^{*}(0)=1$, and $B^{*^{\prime}}(0)=-E(V)$ the mean vacation time, and $F^{*}(0)=1$, and $F^{*^{\prime}}(0)=-E(R)$ is the mean repair time.

Therefore, adding $Q$ to (5.25) and equaling to 1 and simplifying we get

$$
Q=\frac{\alpha\left(1-p(\lambda E(I)-\delta) G^{*}(a) E(V)\right)-\left(1-G^{*}(a)\right)(\lambda E(I)+\alpha\{1+(\lambda E(I)-\delta) E(R)\})}{\alpha\left\{\delta E(R)\left(1-G^{*}(\alpha)\right)+G^{*}(\alpha)(1+p \delta E(V))\right\}} .
$$

From (5.28) we can find the utilization factor, $\rho$, where $\rho=1-Q$.

As a particular case if we assume there is no stand by server this means that $\delta=0$, $b=c=\lambda-\lambda E(I)$ using this in the main results of this paper, we get,

$$
\begin{gathered}
S_{q}(z)=\frac{-Q\left(1-G^{*}(a)\right)\left\{b+\alpha z\left(1-F^{*}(b)\right)\right\}-a Q p G^{*}(a)\left(1-B^{*}(b)\right)}{a\left(z-G^{*}(a)\left(1-p+p B^{*}(b)\right)\right)-\alpha z\left(1-G^{*}(a)\right) F^{*}(b)}, \\
Q=1-\lambda E(I)\left\{\frac{1}{\alpha G^{*}(a)}-\frac{1}{\alpha}+\frac{E(R)}{G^{*}(a)}-E(R)+p E(V)\right\} .
\end{gathered}
$$

These results agree with results given by [9].

\section{The Average Queue Size and the Average Waiting Time}

Let $L_{q}$ denote the mean number of customers in the queue under the steady state. Then

$$
L_{q}=\left.\frac{d}{d z} S_{q}(z)\right|_{z=1}
$$


Since this formula gives $0 / 0$ form, then using the L'Hopital's rule four times we obtain

$$
L_{q}=\lim _{z \rightarrow 1} \frac{D^{\prime \prime}(z) N^{\prime \prime \prime}(z)-N^{\prime \prime}(z) D^{\prime \prime \prime}(z)}{3\left(D^{\prime \prime}(z)\right)^{2}}
$$

where $N^{\prime \prime}(1)$ and $D^{\prime \prime}(1)$ are given in (5.26) and (5.27), respectively, and

$$
\begin{aligned}
N^{\prime \prime \prime}(1)= & -3 Q n y\left(1-G^{*}(\alpha)\right)(1+\alpha E(R)) \\
& -6 Q y m^{2} G^{* \prime}(\alpha)[1+\alpha E(R)] \\
& -3 Q m\left(1-G^{*}(a)\right)\left[x(1+\alpha E(R))+2 \alpha y E(R)+\alpha y^{2} E\left(R^{2}\right)\right] \\
& +3 p Q E(V) y G^{*}(a)\left\{2 m^{2}-\alpha n\right\} \\
& +6 \alpha p Q m^{2} y E(V) G^{* \prime}(a) \\
& -3 \alpha p Q m G^{*}(\alpha)\left(y^{2} E\left(V^{2}\right)+x E(V)\right), \\
D^{\prime \prime \prime}(1)= & 3\left(1-G^{*}(a) p E(V) y\right)\{2 m y-\alpha x\} \\
& +6 m^{2} y G^{* \prime}(\alpha)+3\left(1-G^{*}(a)\right)\{n y+m x\} \\
& -3 \alpha y p\left(2 G^{* \prime}(a) m y E(V)-G^{*}(a)\left(E\left(V^{2}\right) y^{2}+x E(V)\right)\right) \\
& +6 \alpha y^{2}\left(1-G^{*}(a)\right) E(R)+3 \alpha\{1+y E(R)\}\left\{x\left(1-G^{*}(a)\right)+2 m y G^{* \prime}(\alpha)\right\} \\
& +3 \alpha y\left(1-G^{*}(a)\right)\left(y^{2} E\left(R^{2}\right)+x E(R)\right),
\end{aligned}
$$

where $(\lambda E(I(I-1))+2 \delta)=x, \lambda E(I)-\delta=y, \lambda E(I(I-1))=n$, and $\lambda E(I)=m$.

\section{Numerical Example}

To illustrate the results of this chapter numerically we consider that the service times, vacation times, stand-by service times, and repair times are exponentially distributed. All the values were chosen so that the steady state condition is satisfied. In Table 1 we will show the effect of the new contribution of this paper, where we will show the influence of the new parameter $\delta$ (stand-by service rate) on the trends. We choose the following values $\mu=7, \gamma=3, \lambda=2$, $\beta=7, \alpha=2, P=0.5, E(I)=1$, and $E(I(I-1))=0$, we consider that $\delta$ takes the values $0,1,4$, 6,8 , and 10 . 
Table 1: Computed values of various queue performance measures $(\mu=7, \lambda=2, \alpha=2, P=0.5, \gamma=$ $3, \beta=7)$.

\begin{tabular}{lcccccc}
\hline$\delta$ & $\rho$ & $Q$ & $L_{q}$ & $W_{q}$ & $L$ & $W$ \\
\hline 0 & 0.619 & 0.381 & 1.4048 & 0.7024 & 2.0238 & 1.0119 \\
1 & 0.5306 & 0.4694 & 0.8064 & 0.4032 & 1.3371 & 0.6685 \\
4 & 0.3714 & 0.6286 & 0.3432 & 0.1716 & 0.7147 & 0.3573 \\
6 & 0.3095 & 0.6905 & 0.2807 & 0.1403 & 0.5902 & 0.2951 \\
8 & 0.2653 & 0.7347 & 0.2617 & 0.1309 & 0.527 & 0.2635 \\
10 & 0.2321 & 0.7679 & 0.2591 & 0.1295 & 0.4912 & 0.2456 \\
\hline
\end{tabular}

Table 1 shows that increasing the value of $\delta$ decreases the value of utilization factor, the mean queue size, and the mean waiting time of the customers while the server idle time increases. All the trends shown by the table are as expected.

\section{Acknowledgments}

The authors would like to express their deep appreciation and thanks to the referee(s) for their comments and suggestions, which led to improvement of the paper in its present form.

\section{References}

[1] Y. Levy and U. Yechiali, "An M/M/s queue with servers vacations," INFOR Journal, vol. 14, no. 2, pp. 153-163, 1976.

[2] S. W. Fuhrmann and R. B. Cooper, "Stochastic decomposition in $M / G / 1$ queue with generalized vacations," Operations Research, vol. 33, no. 5, pp. 1117-1129, 1985.

[3] B. T. Doshi, "Queueing systems with vacations-a survey," Queueing Systems, vol. 1, no. 1, pp. 29-66, 1986.

[4] A. Aissani and J. R. Artalejo, "On the single server retrial queue subject to breakdowns," Queueing Systems, vol. 30, no. 3-4, pp. 309-321, 1998.

[5] J. Wang, J. Cao, and Q. Li, "Reliability analysis of the retrial queue with server breakdowns and repairs," Queueing Systems, vol. 38, no. 4, pp. 363-380, 2001.

[6] W. J. Gray, P. P. Wang, and M. Scott, "A queueing model with multiple types of server breakdowns," Quality Technology and Quantitative Management, vol. 1, no. 2, pp. 245-255, 2004.

[7] Q. Xu, S. Bao, Z. Ma, and N. Tian, " $M^{[X]} / G / 1$ queue with multiple vacations," Stochastic Analysis and Applications, vol. 25, no. 1, pp. 127-140, 2007.

[8] K. C. Madan, W. Abu-Dayyeh, and M. Gharaibeh, "Steady state analysis of two $M x / M a, b / 1$ queue models with random breakdowns," International Journal of Information and Management Sciences, vol. 14, no. 3, pp. 37-51, 2003.

[9] F. A. Maraghi, K. C. Madan, and K. Darby-Dowman, "'Batch arrival queueing system with random breakdowns and Bernoulli schedule server vacations having general vacation time distribution," International Journal of Information and Management Sciences, vol. 20, no. 1, pp. 55-70, 2009.

[10] C. K. Madan, "A bulk input queue with a stand-by," The South African Statistical Journal, vol. 29, pp. 1-7, 1995. 


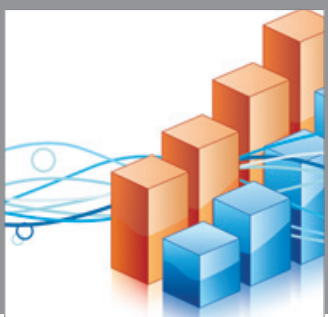

Advances in

Operations Research

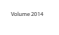

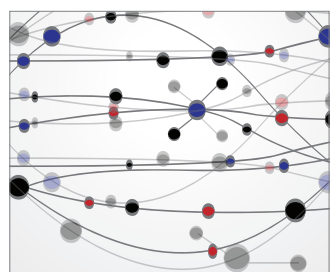

\section{The Scientific} World Journal
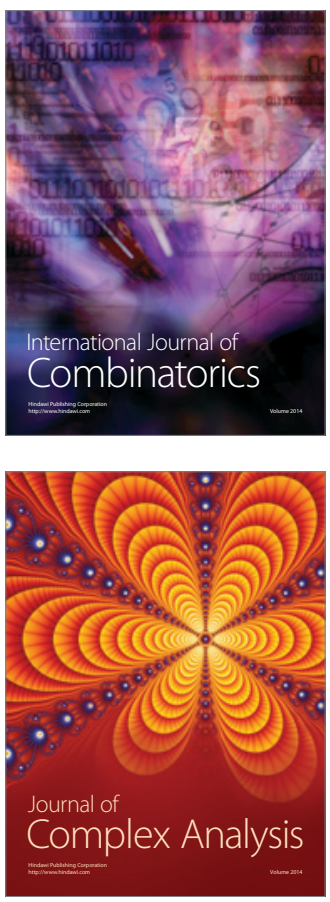

International Journal of

Mathematics and

Mathematical

Sciences
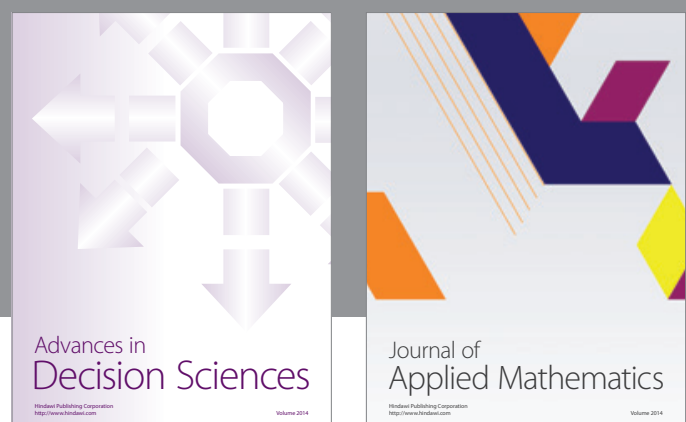

Journal of

Applied Mathematics
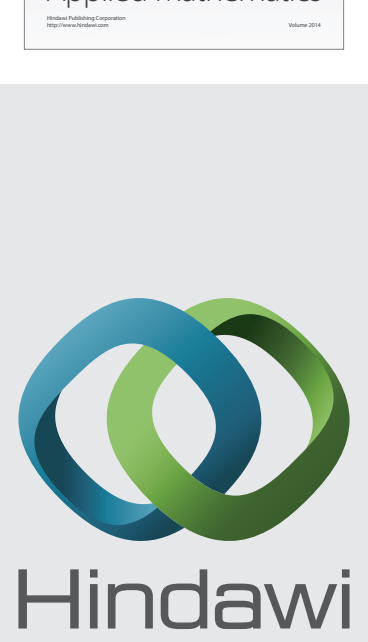

Submit your manuscripts at http://www.hindawi.com
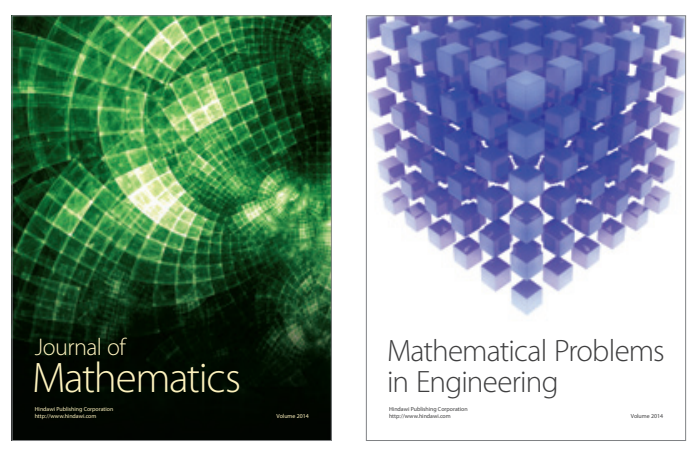

Mathematical Problems in Engineering
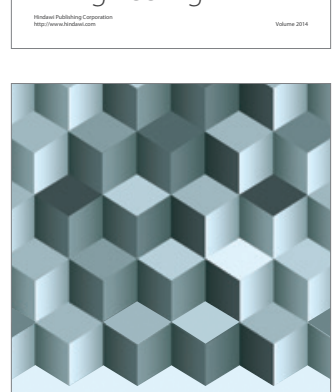

Journal of

Function Spaces
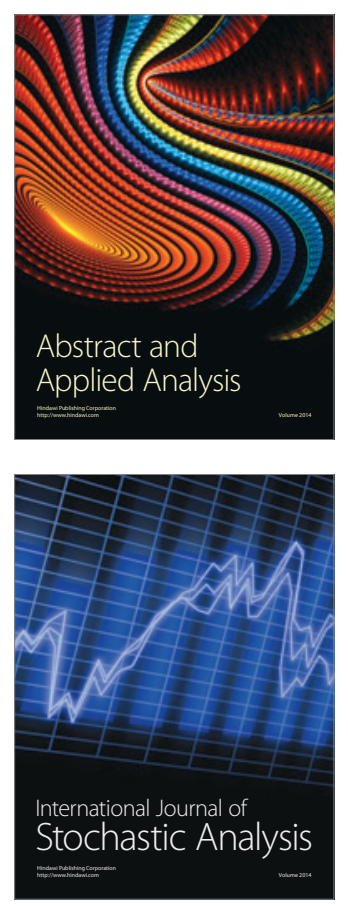

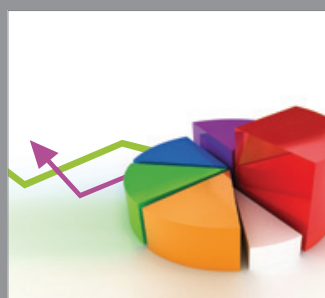

ournal of

Probability and Statistics

Promensencen
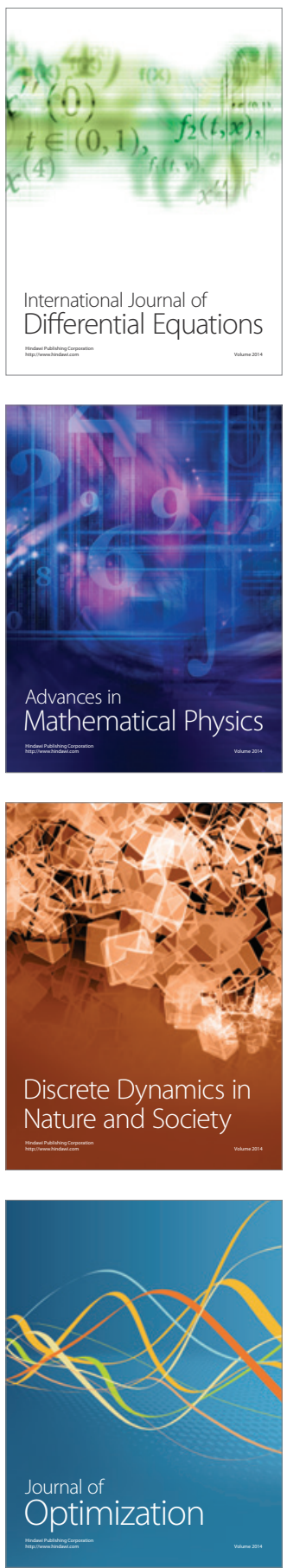